J. Lake Sci. (湖泊科学), $2007, \mathbf{1 9 ( 3 ) ~ : 2 2 7 - 2 3 4 ~}$

http:// www. jlakes. org. E-mail: jlakes@ niglas. ac.cn

(c) 2007 by Journal of Lake Sciences

\title{
太湖秋季水体遥感反射比的简单经验估测模型
}

\author{
马荣华 ${ }^{1,2}$, 宋庆君 $^{3}$, 唐军武 ${ }^{3}$, 潘德炉 $^{2}$ \\ (1: 中国科学院南京地理与湖泊研究所, 南京 210008) \\ (2: 卫星海洋环境动力学国家重点实验室, 国家海洋局第二海洋研究所, 杭州 310012) \\ (3: 国家卫星海洋应用中心, 北京 100081)
}

摘 要:2004 年 10 月现场测量了太湖水体的遥感反射比、后向散射系数以及其他必要参数,实验室测量了水体的悬浮颗 粒物质浓度. 首先把太湖划分为 3 个大区, 即遥感反射比受湖底影响的区域 (影响区)、不受湖底影响的区域 (非影响区) 以及可能受湖底影响的区域 (可能影响区), 然后再把太湖分为高混浊、中混浊、低清澈以及高清澈等 4 类水体类型, 分别 列出了各种水体区域类型的相关属性数据. 在整个太湖区域范围内,建立了后向散射和悬浮颗粒物质浓度的经验回归模 型; 仅在非影响区内, 建立了遥感反射比和后向散射的经验回归模型,并可以利用悬浮颗粒物质浓度直接估测遥感反射 比. 最后详细分析了模型的精度.

关键词:遥感反射比;后向散射; 悬浮颗粒; 太湖

\section{A simple empirical model for remote sensing reflectance of Lake Taihu waters in autumn}

MA Ronghua ${ }^{1,2}$, SONG Qingjun ${ }^{3}$, TANG Junwu ${ }^{3} \&$ PAN Delu ${ }^{2}$

(1:Nanjing Institute of Geography and Limnology, CAS, Nanjing 210008, P. R. China)

(2: State Key Laboratory of Satellite Ocean Environment Dynamics, Second Institute of Oceanography, State Oceanic Administration, Hangzhou 310012, P. R. China)

(3: National Satellite Ocean Application Service, Beijing 100081, P. R. China)

Abstract: Remote sensing reflectance, backscattering and other corresponding parameters were measured in situ in Lake Taihu mainly following with NASA protocols. And suspended particulate matter ( SPM) concentrations were measured in the lab mainly following with lake investigation criterion of China. Firstly, the whole lake was divided into three areas based on the literature's results, where the remote sensing reflectance was influenced, not influenced, and possibly influenced by the lake bottom, respectively. They were named by the influenced area, the non-influenced area, and the possibly influenced area, respectively. Then, we divided the whole waters into two types and four sub-types based on the Secchi disk transparency measured in situ, viz. the turbid water, including the high-turbid water and the mid-turbid water, and the clear water, including the low-clear water and the highclear water. And their properties were described by the corresponding data measured in situ or in the lab. In the whole lake, the empirical model was developed between the backscattering coefficient and SPM concentration. Only in the non-influenced area was developed the empirical model between the pairs of the remote sensing reflectance and backscattering coefficient. The remote sensing reflectance can be estimated approximately by SPM concentration and the estimation models were developed based on the mentioned above. Finally, the model precisions were analyzed in detail. Maybe it is a very good approach to preliminarily know about apparent optical properties of waters in Lake Taihu when there are not optical data measured in situ but only SPM concentrations measured in the lab.

Keywords: Remote sensing reflectance; backscattering; suspended particulate matter; Lake Taihu

* 国家自然科学基金资助 (40671138).2006-06-22 收稿;2006-09-24 收修改稿. 马荣华,男,1972 年生,博士,副 研究员;E-mail:rhma@ niglas. ac. cn. 
水色/水质遥感中, 遥感反射比 (remote sensing reflectance) 是最重要的一个表观光学量, 是生物光学模 型中必不可少的物理量,隐含有水体物质组分含量信息. 因此,遥感反射比的现场测量 (方法、精度) 与定量 估测一直受到水色遥感研究领域的高度重视. 在现场测量方面,已经比较成熟,包括测量使用的仪器、测量 方法与步骤以及测量后的数据处理等 ${ }^{[1]}$. 定量估测方法主要有两种: (1) 通过卫星遥感影像来进行, 伴随着 遥感正演过程; (2) 通过生物光学模型来进行,伴随着遥感反演过程. 这两种方法都有着较为坚实的理论基 础, 但需要大量其他相关数据的配合. 与海洋 I 类水体相比,太湖水体物质组分复杂,一方面表现为影响水 体光学特性的物质组成的种类多,包括浮游植物色素、有机悬浮颗粒、无机悬浮颗粒、黄色物质等,另一方面 表现为这些物质的来源复杂, 有陆源的, 有自身降解的, 有湖底再悬浮的, 另外还表现为物质颗粒属性(大 小、形状以及内部结构等) 的差异以及颗粒空间分布的差异. 目前在悬浮颗粒物质浓度与后向散射系数的 关系方面研究较多 ${ }^{[2]}$, 但在悬浮颗粒物质浓度与遥感反射比的定量估测方面, 鲜有报道. 本文试图通过悬 浮颗粒物质浓度、后向散射系数以及遥感反射比的关系分析, 建立一个理论依据较为充分的遥感反射比的 简单经验回归估测模型, 以服务于太湖水色遥感反演.

\section{1 方法}

\section{1 试验量测}

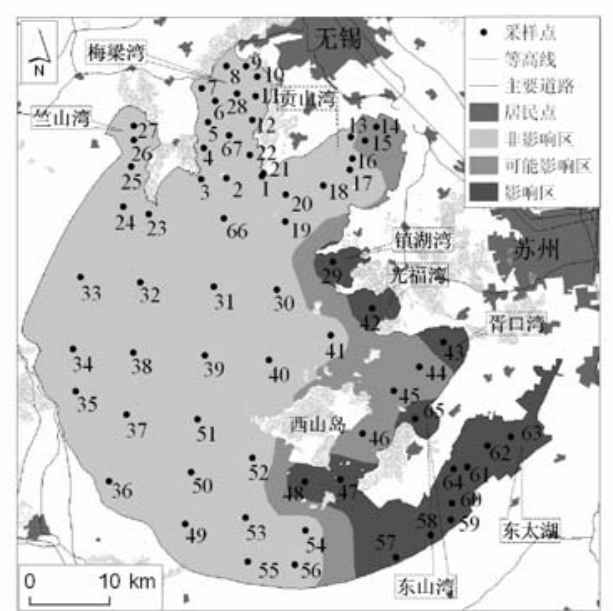

图 1 样点位置及湖底对遥感反射比的 影响区域分布

Fig. 1 Spatial distribution of 67 samples in Lake Taihu

根据太湖的平均水质状况, 共布置了 67 个样点 (图 1 ), 这些样点几乎覆盖了太湖的整个水域,水质变化从高 度富营养化区 (梅梁湾) 到中富营养化区 (东太湖), 从湖 边到湖心. 依据 NASA SeaWiFS 海洋光学规范, 采用 $\left[40^{\circ}\right.$, $\left.135^{\circ}\right]$ 的测量几何以及水面以上测量法 ${ }^{[1]}$, 通过双通道地 物波谱仪 FieldSpec 931(ASD 公司, 光谱范围为 350-1050 $\mathrm{nm}$, 光纤视场角为 $25^{\circ}$, 加前置镜头后视场角为 $8^{\circ}$, 对两个 辐亮度探测器进行了绝对辐射定标, 定标结果符合规范要 求), 获得了 66 个样点的遥感反射比 $R_{r s}$ (由于天气原因, 48 号点没有进行遥感反射比测量); 使用 HS -6 (HydroScat - 6 Spectral Backscattering Sensor) 分别在 442、 488、532、589、676 以及 $852 \mathrm{~nm}$ 处现场测量水体的总后向 反射系数,经校正后获得最终的 6 波段后向散射系数 $b_{b}$. 实验室内测量水体的物质含量浓度,包括叶绿素 a 浓度 $C_{C H L}$ 、悬浮物含量浓度 $C_{S P M}$ ( = 有机悬浮物含量浓度 $C_{S P O M}$ + 无机悬浮物含量浓度 $C_{S P I M}$ )、DOC 含量浓度 $C_{D O C}$; 另外, 现场测量了水深 $H$ 以及水体的白色塞氏圆盘透明度 $S$. 有 关参数的详细测量过程详见文献 $[3]$ 和 $[4]$.

\section{2 数据分析}

假设遥感反射比的测量误差为 0 ,则有:

$$
R_{r s}^{m}=R_{r s}^{b}+R_{r s}^{w}
$$

式中, $R_{r s}^{m}$ 表示实测遥感反射比, $R_{r s}^{b}$ 表示来自湖底的遥感反射比, $R_{r s}^{w}$ 表示仅来自水体的遥感反射比.

对于光学浅水湖泊, 特别是湖深小于 3 倍透明度的水体而言, 底质对离水辐射的影响较为明显 ${ }^{[5]}$. 据 此首先把 67 个样点分为两类, 一类是遥感反射比受湖底影响的样点 (属性赋值为 1 ), 另一类是遥感反射比 不受湖底影响的样点 (属性赋值为 0 ), 然后使用 IDW ( inverse distance weighted) 插值到整个湖区, 把太湖水 体划分为遥感反射比受湖底影响的区域 (属性值大于 0.6 )、不受湖底影响的区域( 属性值小于 0.2 ) 以及可 能受湖底影响的区域 (属性值在 0.2-0.6 之间), 分别简称为影响区、非影响区和可能影响区 (图 1 ), 详细

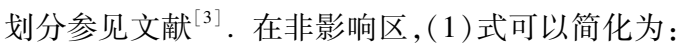

$$
R_{r s}^{m}=R_{r s}^{w}
$$


另外, 不同水质的水体中, 物质组成有很大差别, 可能会 造成不同的遥感反射比估测模式 (模型). 因此对于水质差别 很大的太湖水体, 为了提高遥感反射比的估测精度, 根据水质 进行水体分区是必要的. 水质在一定程度上影响着水体的后 向散射, 透明度是水体浑浊度和水质的重要指标. 在太湖, 透 明度 $S(\mathrm{~cm})$ 和悬浮物含量浓度 $C_{S P M}(\mathrm{mg} / \mathrm{L})$ 之间存在着显著 的负指数关系, 如式 (3), 据此我们可以根据透明度把整个太 湖水体分成 4 类 (图 2): (1) 高浑浊水体 (透明度小于 30 $\mathrm{cm}),(2)$ 中浑浊水体 (透明度在 $30-60 \mathrm{~cm}$ ), (3) 低清澈水体 (透明度在 $60-100 \mathrm{~cm}$ ), (4) 高清澈水体 (透明度大于 100 $\mathrm{cm})$; 其中高浑浊水体和中浑浊水体总称浑浊水体, 低清澈水 体和高清澈水体总称清澈水体.

$$
C_{S P M}=15200 \times S^{-1.6302},\left(R^{2}=0.9392, N=67\right)
$$

为了和后向散射系数的 6 个波段相对应, 以 $442 、 488$ 、 $532 、 589 、 676$ 和 $852 \mathrm{~nm}$ 为中心,上下各 $5 \mathrm{~nm}$ 为范围, 分别对 实测遥感反射比 $R_{r s}^{m}$ 求平均, 作为上述 6 个波段的遥感反射

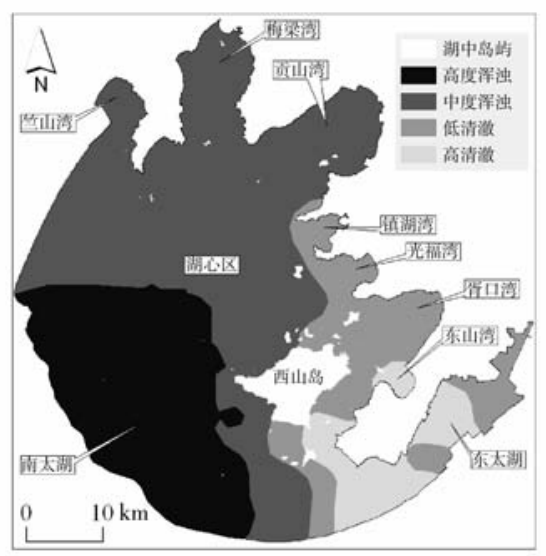

图 2 四种混浊程度水体的空间分布

Fig. 2 Spatial distribution of four waters with different turbidities 比. 然后在不同的水质类型区, 通过常用参数 (如悬浮物浓度), 利用线性或非线性回归法, 分别构建遥感反 射比的简单估测模型.

\section{2 结果与分析}

表 1 列出了不同类型水体 (高混浊水体、中 混浊水体、低清澈水体以及高清澈水体,非影响 区水体、影响区水体以及可能影响区水体) 的透 明度、悬浮物含量浓度、无机悬浮颗粒浓度、6 波 段后向散射系数以及 6 波段遥感反射比的数值 范围. 图 3 给出了太湖水体部分样点的实测遥 感反射比曲线. 图 4 给出了 4 类水体的部分样 点的后向散射曲线, 以表明不同水体后向散射系 数之间的差别.

\section{1 简单经验模型}

图 5 表示非影响区内不同类型水体的遥感

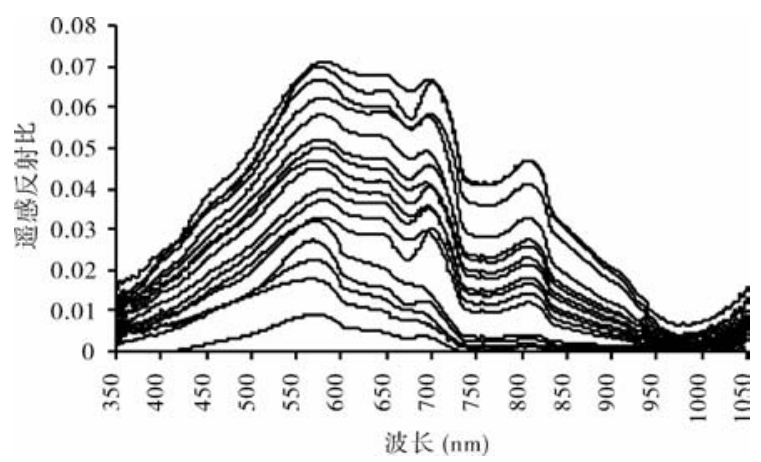

图 3 太湖水体部分样点的实测遥感反射比

Fig. 3 The remote sensing reflectance measured in situ 反射比和后向散射系数之间的相关关系随波长的变化; 在整个波长范围内,高浑浊水体表现出明显的差别, 仅在 $852 \mathrm{~nm}$ 处存在显著的相关关系 (相关系数 $R$ 大于 0.8 ), 而其它类型的水体在整个波长范围内都存在 这种显著的相关关系. 因此, 在非影响区内 (剔除 17 号点和 21 号点), 通过后向散射系数估测遥感反射比 是可能的. 利用各种线性或非线性模型进行回归拟合分析,结果表明,在各个波段,存在下述最好的回归 关系:

$$
\begin{aligned}
& R_{r s}(442)=0.0184 \times b_{b}(442)+0.0025,\left(R^{2}=0.7838, N=46\right) \\
& R_{r s}(488)=0.0309 \times b_{b}(488)+0.0042,\left(R^{2}=0.7507, N=46\right) \\
& R_{r s}(532)=0.0280 \times b_{b}(532)+0.0144,\left(R^{2}=0.7378, N=46\right) \\
& R_{r s}(589)=0.0303 \times b_{b}(589)+0.0200,\left(R^{2}=0.7630, N=46\right) \\
& R_{r s}(676)=0.0229 \times b_{b}(676)+0.0130,\left(R^{2}=0.9049, N=46\right) \\
& R_{r s}(852)=0.0117 \times b_{b}(852)-0.0004,\left(R^{2}=0.9601, N=46\right)
\end{aligned}
$$

上述方程可以用形如 $(9)$ 式的模型进行统一表示:

$$
R_{r s}(\lambda)=K_{b} \times b_{b}(\lambda)+B_{b},\left(R^{2}=>0.73\right)
$$


式中, $K_{b}$ 和 $B_{b}$ 是常数, 可以通过形如 (3) - (8) 的回归方程来获取.

表 1 太湖不同类型水体的一些属性

Tab. 1 Some properties of different waters in Lake Taihu

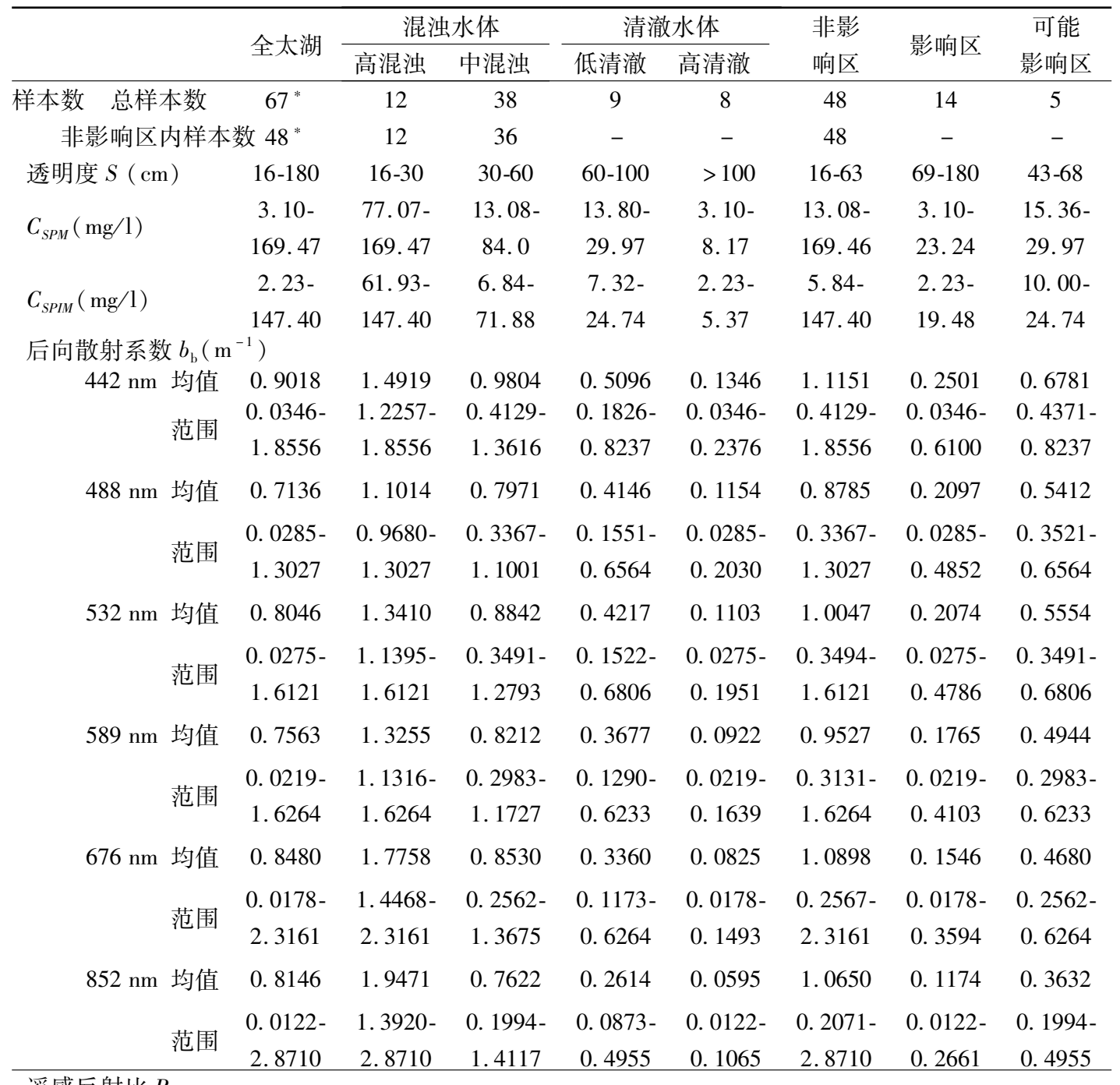

遥感反射比 $R_{\mathrm{rs}}$

\begin{tabular}{|c|c|c|c|c|c|c|c|c|c|}
\hline \multirow[t]{2}{*}{$442 \mathrm{~nm}$} & 均值 & 0.0211 & 0.0311 & 0.0201 & 0.0160 & 0.0082 & 0.0243 & 0.0096 & 0.0195 \\
\hline & 艺围 & $\begin{array}{c}0.0012- \\
0.0789\end{array}$ & $\begin{array}{l}0.0267- \\
0.0351\end{array}$ & $\begin{array}{c}0.0102- \\
0.0312\end{array}$ & $\begin{array}{c}0.0070- \\
0.0238\end{array}$ & $\begin{array}{c}0.0012- \\
0.0128\end{array}$ & $\begin{array}{c}0.0102- \\
0.0789\end{array}$ & $\begin{array}{l}0.0012- \\
0.0161\end{array}$ & $\begin{array}{c}0.0114- \\
0.0238\end{array}$ \\
\hline \multirow[t]{2}{*}{$488 \mathrm{~nm}$} & 均值 & 0.0287 & 0.0403 & 0.0281 & 0.0227 & 0.0123 & 0.0327 & 0.0142 & 0.0278 \\
\hline & 池沏 & $\begin{array}{c}0.0035- \\
0.0890\end{array}$ & $\begin{array}{l}0.0351- \\
0.0459\end{array}$ & $\begin{array}{c}0.0161- \\
0.0410\end{array}$ & $\begin{array}{c}0.0108- \\
0.0335\end{array}$ & $\begin{array}{c}0.0035- \\
0.0195\end{array}$ & $\begin{array}{c}0.0161- \\
0.0890\end{array}$ & $\begin{array}{c}0.0035- \\
0.0230\end{array}$ & $\begin{array}{c}0.0177- \\
0.0335\end{array}$ \\
\hline \multirow[t]{2}{*}{$532 \mathrm{~nm}$} & 均值 & 0.0391 & 0.0536 & 0.0388 & 0.0326 & 0.0178 & 0.0441 & 0.0207 & 0.0388 \\
\hline & & $\begin{array}{c}0.0067- \\
0.0954\end{array}$ & $\begin{array}{c}0.0454- \\
0.0608\end{array}$ & $\begin{array}{c}0.0241- \\
0.0552\end{array}$ & $\begin{array}{c}0.0183- \\
0.0474\end{array}$ & $\begin{array}{c}0.0067- \\
0.0276\end{array}$ & $\begin{array}{l}0.0241- \\
0.0954\end{array}$ & $\begin{array}{c}0.0067- \\
0.0323\end{array}$ & $\begin{array}{l}0.0264- \\
0.0474\end{array}$ \\
\hline
\end{tabular}




\begin{tabular}{|c|c|c|c|c|c|c|c|c|c|}
\hline \multirow[t]{2}{*}{$589 \mathrm{~nm}$} & 均值 & 0.0443 & 0.0622 & 0.0443 & 0.0360 & 0.0178 & 0.0502 & 0.0225 & 0.0440 \\
\hline & 范围 & $\begin{array}{c}0.0080- \\
0.0948\end{array}$ & $\begin{array}{c}0.0523- \\
0.0708\end{array}$ & $\begin{array}{c}0.0298- \\
0.0629\end{array}$ & $\begin{array}{l}0.0229- \\
0.0525\end{array}$ & $\begin{array}{c}0.0080- \\
0.0294\end{array}$ & $\begin{array}{c}0.0298- \\
0.0948\end{array}$ & $\begin{array}{c}0.0080- \\
0.0348\end{array}$ & $\begin{array}{r}0.0310- \\
0.0525\end{array}$ \\
\hline \multirow[t]{2}{*}{$676 \mathrm{~nm}$} & 均值 & 0.0324 & 0.0541 & 0.0323 & 0.0224 & 0.0099 & 0.0382 & 0.0122 & 0. 0297 \\
\hline & 范围 & $\begin{array}{c}0.0034- \\
0.0640\end{array}$ & $\begin{array}{l}0.0455- \\
0.0640\end{array}$ & $\begin{array}{c}0.0179- \\
0.0504\end{array}$ & $\begin{array}{c}0.0109- \\
0.0353\end{array}$ & $\begin{array}{c}0.0034- \\
0.0165\end{array}$ & $\begin{array}{c}0.0183- \\
0.0640\end{array}$ & $\begin{array}{c}0.0034- \\
0.0230\end{array}$ & $\begin{array}{c}0.0179- \\
0.0353\end{array}$ \\
\hline \multirow[t]{2}{*}{$852 \mathrm{~nm}$} & 均值 & 0.0097 & 0.230 & 0.0087 & 0.0031 & 0.0010 & 0.0125 & 0.0015 & 0.0043 \\
\hline & 范围 & $\begin{array}{c}0.0000- \\
0.0308\end{array}$ & $\begin{array}{c}0.0163- \\
0.0308\end{array}$ & $\begin{array}{l}0.0022- \\
0.0213\end{array}$ & $\begin{array}{l}0.0013- \\
0.0057\end{array}$ & $\begin{array}{l}0.0000- \\
0.0021\end{array}$ & $\begin{array}{l}0.0022- \\
0.0308\end{array}$ & $\begin{array}{l}0.0000- \\
0.0036\end{array}$ & $\begin{array}{l}0.0028- \\
0.0060\end{array}$ \\
\hline
\end{tabular}

* 由于天气原因,48 号点 (属清澈水体) 没有测量遥感反射比; 17 号点 (非影响区、中混浊水体) 遥感反 射比曲线异常, 21 号点 (非影响区、中混浊水体) 后向散射系数曲线异常. 视不同观测量的分析, 剔除上述 异常点数据.
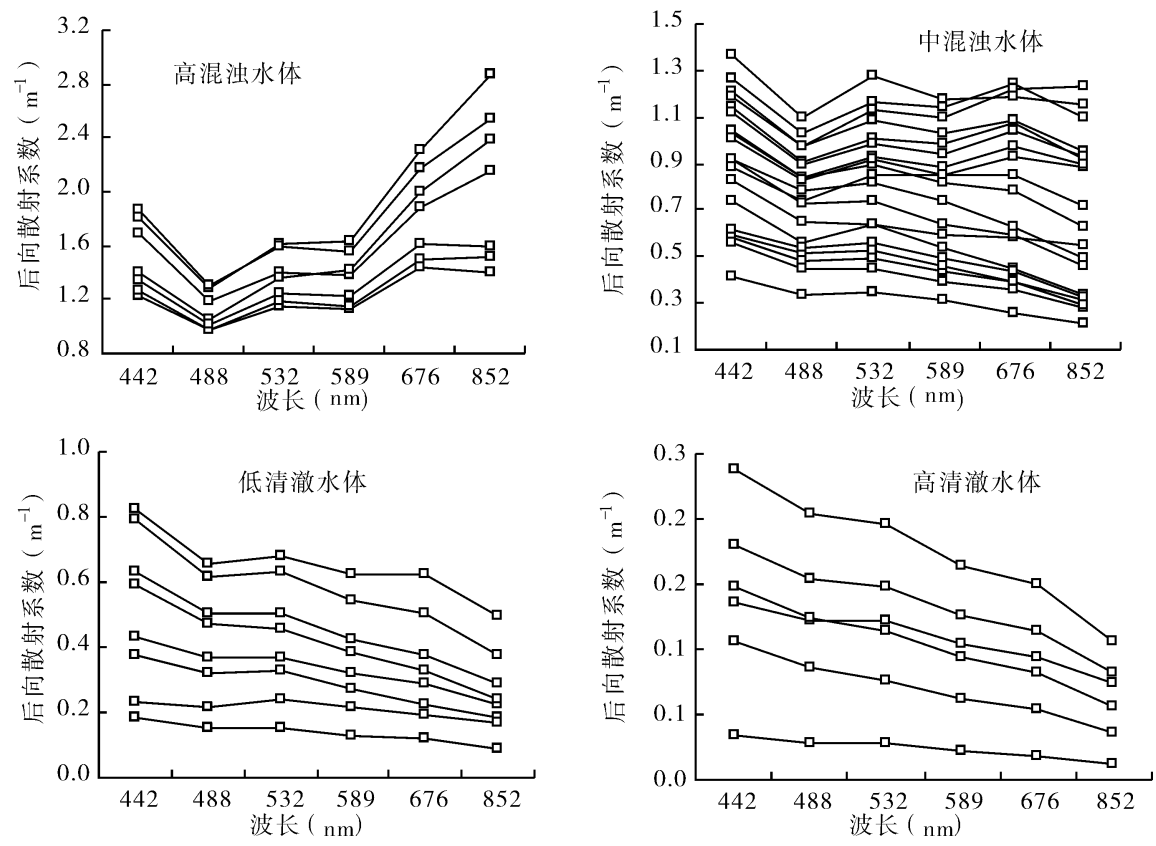

图 4 太湖不同水质类型水体的后向散射系数

Fig. 4 backscattering coefficient measured in situ in different types of waters

不同类型水体的后向散射系数与无机悬浮颗粒浓度以及有机悬浮颗粒浓度之间的关系分析表明,无机 悬浮颗粒基本上主导着水体的后向散射,但有机悬浮颗粒的贡献并不能总是被忽略. 后向散射系数和总悬 浮颗粒的关系随波长的变化与后向散射系数和无机悬浮颗粒的关系随波长的变化是一致的,但前者的相关 度 (相关系数) 总是大于后者,二者的相关分析表明: (1) 对浑浊水体以及整个太湖水体而言, 在 $852 \mathrm{~nm}$ 处, 在后向散射系数与总悬浮颗粒浓度以及无机悬浮颗粒浓度之间存在着显著的形如 (10) 式的线性关系 $\left(R_{S P M}^{2}>R_{S P I M}^{2}>0.90\right)$, 但在 $442 、 488 、 532 、 589$ 以及 $676 \mathrm{~nm}$ 处,最好的回归关系是形如(11) 式的对数模型 $\left(R_{S P M}^{2}>R_{S P I M}^{2}>0.82\right)$; (2) 对清澈水体而言,在上述 6 个波段处, 后向散射系数与总悬浮颗粒浓度以及无 机悬浮颗粒浓度之间都可以用形如 (10) 式的线性模型来表示.

$$
\begin{aligned}
& b_{b}(\lambda)=K_{C 1} \times C_{i}+B_{C 1}, R^{2}>0.90, \mathrm{~m}^{-1} \\
& b_{b}(\lambda)=K_{C 2} \times \ln \left(C_{i}\right)+B_{C 2}, R^{2}>0.82, \mathrm{~m}^{-1}
\end{aligned}
$$




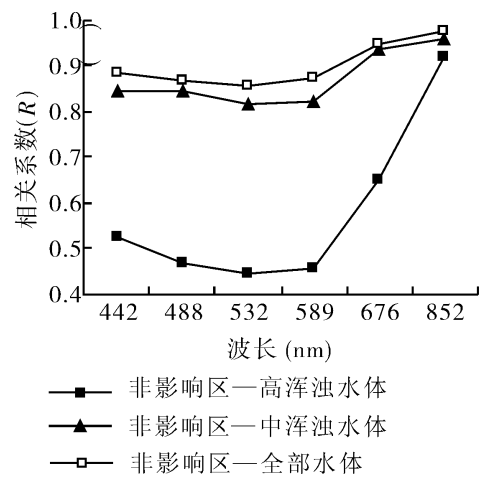

图 5 非影响区内后向散射系数与 遥感反射比之间的相关关系

Fig. 5 Correlation relationships of pairs of backscattering coefficients and remote sensing reflectance measured in situ in the noninfluenced area: comparisons between different wavelengths and the variation tendencies following with wavelength

式中, $i(=1,2)$ 分别表示 SPM 和 SPIM. $K_{C 1}$ 和 $B_{C 1} 、 K_{C 2}$ 和 $B_{C 2}$ 能够 通过图 6 所示的回归方程来获取.

\section{2 模型精度}

假设悬浮颗粒物质浓度 $C_{i}$ 、后向散射系数 $b_{b}$ 以及遥感反射比 $R_{r s}$ 等观测量的测量误差为 0 , 通过 $C_{i}$ 估测 $b_{b}$ 的绝对误差为 $e_{b}$ ，相对 误差为 $e_{b r}$; 通过 $b_{b}$ 估测 $R_{r s}$ 的绝对误差为 $e_{R}$ 、相对误差为 $e_{R r}$; 通过 $C_{i}$ 估测 $R_{r s}$ 的绝对误差为 $e_{t}$ 、相对误差为 $e_{t r}$; 根据误差传播定律, 有:

$e_{1}=e_{R}+K_{b} \times e_{b}$

各自的平均误差可以表示为:

$$
\begin{aligned}
& \overline{e_{b}}=\sum_{i=1}^{N} e_{b i} / N, \overline{e_{b r}}=\sum_{i=1}^{N} e_{b r_{i}} / N, \overline{e_{R}}=\sum_{i=1}^{N} e_{R_{i}} / N, \\
& \overline{e_{R r}}=\sum_{i=1}^{N} e_{R_{r_{i}}} / N, \overline{e_{t}}=\sum_{i=1}^{N} e_{t_{i}} / N, \overline{e_{\mathrm{tr}}}=\sum_{i=1}^{N} e_{t r_{i}} / N,
\end{aligned}
$$

式中, $N$ 为参与计算的样点数, $i$ 为采样点序号.

表 2 表明,非影响区内,通过悬浮颗粒物质浓度估测后向散射 系数的相对估测误差平均约为 $11.8 \%$, 最小的位于 $589 \mathrm{~nm}$ 处, 约 $9.4 \%$,最大的位于 $676 \mathrm{~nm}$ 处,约 $14.5 \%$, 红外近红外波段的估测误 差明显大于其他可见光波段; 直接通过后向散射系数估测遥感反 射比的相对估测误差平均约为 $10.7 \%$,最小的位于 $676 \mathrm{~nm}$ 处,约为 $8.3 \%$, 最大的位于 $852 \mathrm{~nm}$ 处,约为 $12.7 \%$; 通过悬浮物浓度估测遥 感反射比的相对估测误差平均约为 $19.2 \%$,最小的位于 $589 \mathrm{~nm}$ 处,约为 $14.4 \%$,最大的位于 $852 \mathrm{~nm}$ 处,约 为 $25.7 \%$.

表 2 非影响区内水体不同波段处的后向散射系数估测误差以及遥感反射比估测误差

Tab. 2 Estimation errors of backscattering coefficient and remote sensing reflectance for waters in non-influenced area

\begin{tabular}{lccccccc}
\hline & $442 \mathrm{~nm}$ & $488 \mathrm{~nm}$ & $532 \mathrm{~nm}$ & $589 \mathrm{~nm}$ & $676 \mathrm{~nm}$ & $852 \mathrm{~nm}$ & 平均 \\
\hline$e_{b}\left(\mathrm{~m}^{-1}\right)$ & 0.1136 & 0.0858 & 0.0882 & 0.0725 & 0.1053 & 0.0980 & 0.0939 \\
$e_{b r}(\%)$ & 11.2 & 11.4 & 10.5 & 9.4 & 14.5 & 14.0 & 11.8 \\
$e_{R}$ & 0.0026 & 0.0033 & 0.0041 & 0.0044 & 0.0031 & 0.0012 & 0.0031 \\
$e_{R r}(\%)$ & 12.3 & 11.3 & 10.3 & 9.4 & 8.3 & 12.7 & 10.7 \\
$e_{t}$ & 0.0048 & 0.0060 & 0.0066 & 0.0067 & 0.0055 & 0.0024 & 0.0053 \\
$e_{t r}(\%)$ & 22.3 & 20.5 & 16.6 & 14.4 & 15.6 & 25.7 & 19.2 \\
\hline
\end{tabular}

\section{3 讨论与结论}

在水色/水质遥感中,无论是正演还是反演,遥感反射比都是必不可少的物理量. 遥感反射比属于表观 光学量的范畴,表观光学量和固有光学量是一个有机整体,生物光学模型建立了二者联系的定量模型, 使得 遥感反射比与水体的吸收和后向散射有了相互推演的基础. 在太湖的全部非影响区水体内,最适合的生物 光学模型是 ${ }^{[3]}$ :

$$
R_{r s}(\lambda)=f\left(\frac{b_{b}(\lambda)}{a(\lambda)+b_{b}(\lambda)}\right)
$$

式中, $f$ 可以表示为波长的二次函数,随波长而变化 $(0.0701-0.1064)$, 整个波长范围内均值约为 0.0924 ; 另外, 随着水体水质的不同, $f$ 也呈现出一定的变化, 对非影响区的中混浊水体而言, 随波长的变化范围为 $0.0723-0.1121$, 整个波长范围内均值约为 0.0965 . 

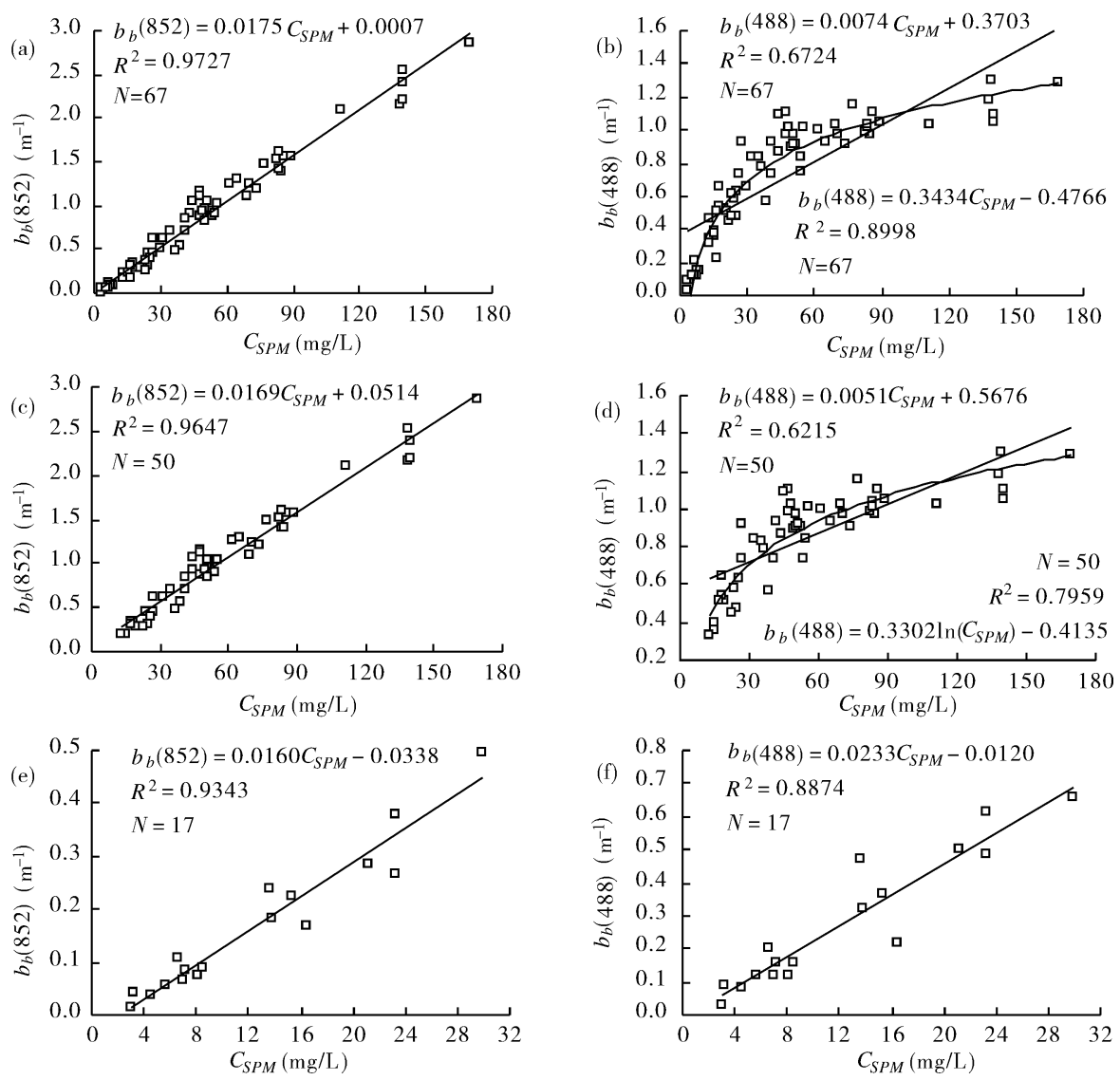

图 $6852 \mathrm{~nm}$ 以及 $488 \mathrm{~nm}$ 处, 后向散射系数 $b_{b}$ 与总悬浮颗粒浓度 $C_{S P M}$ 之间的回归关系:

(a) 和 (b) 太湖整个水体, (c) 和 (d) 浑浊水体, (e) 和 (f) 清澈水体

Fig. 6 Scatter plots of pairs $C_{S P M}$ and $b_{b}$, respectively, at 852 and $488 \mathrm{~nm}$, and their regressions;

(a) and (b), respectively, at 852 and $488 \mathrm{~nm}$ in the whole water, (c) and (d), respectively, at 852 and $488 \mathrm{~nm}$ in the turbid water, (e) and (f) respectively, at 852 and $488 \mathrm{~nm}$ in the clear water

根据 (14) 式,使用实测的后向散射系数和吸收系数(实测的总吸收系数的有效光谱范围 $400-700$ $\mathrm{nm}^{[4]}$ ), 计算整个非影响区内水体在 442、488、532、589 以及 $676 \mathrm{~nm}$ 处的遥感反射比 (简称模型估测), 结果 表明其相对误差随着波长的增加而不断降低, 分别为 $25.0 \% 、 25.0 \% 、 18.6 \% 、 16.0 \%$ 和 $12.2 \%$, 平均 $19.4 \%$; 与表 2 的对比分析表明,基于悬浮颗粒浓度的遥感反射比估测 (简称经验估测) 精度在蓝绿波段 (442、488、532 和 $589 \mathrm{~nm}$ ) 均好于模型估测,仅在红光波段 $676 \mathrm{~nm}$ 处约差 $3.4 \%$, 表明经验估测模型的适用 性, 具有一定的实用价值. 本次实测表明太湖水体的悬浮物质以无机悬浮颗粒为主,约占总悬浮颗粒物质 浓度的 76\% ; 悬浮颗粒的散射作用使水体的遥感反射比在全部可见光和近红外波段范围内都有所增大 ${ }^{[6]}$, 主导着水体的后向散射, 因此通过悬浮物质浓度可以较高精度地估测水体的后向散射系数. 太湖水体的后 向散射又主导着水体的固有光学特性 ${ }^{[3]}$, 因此通过后向散射系数可以较高精度地估测水体的遥感反射比. 统计估测模型的获取过程中, 没有考虑实测数据的现场测量误差. 实际上, 三者 (悬浮物质浓度、后向散射 系数以及遥感反射比) 的测量误差客观存在, 因此提高经验模型参数的观测质量可以提高模型的估测 精度.

太湖非影响区内水体的遥感反射比可以通过悬浮颗粒物质浓度利用线性和/或对数函数模型来估测. 首先,通过(10)式(适用于近红外波段)或(11) 式(适用于可见光波段)估测水体的后向散射系数,估测误 
差在 $12 \%$ 左右, 然后通过(9)式估测水体的遥感反射比,估测的总误差在 $19 \%$ 左右. 在影响区和可能影响 区内, 现场测量的遥感反射比受底质反射的影响, 尚不能建立类似非影响区内的经验估测模型. 但可以利 用已经建立的模型估测影响区和可能影响区内的遥感反射比, 对比现场实测数据, 从而可以大致评估这些 区域内底质反射对遥感反射比的影响. 因此文中模型的应用主要集中在三个方面: (1) 已知悬浮颗粒物质 浓度时,估测全部水体的总后向散射系数以及非影响区水体的遥感反射比; (2) 已知遥感反射比时, 估测非 影响区水体的总后向散射系数以及悬浮颗粒物质浓度; (3) 已知实测遥感反射比以及悬浮颗粒物质浓度 时, 大致估算底质对遥感反射比的贡献. 值得注意的是, 文中的模型基于太湖秋季水体的实测数据, 未必具 有普适性, 特别对太湖而言, 物质含量和组成在不同的季节会表现出较为明显的差别, 因此特别在其它季 节,模型的适用性尚需进一步验证.

致谢: 试验过程中, 得到了李铜基、孙小洲、高飞、张运林、杨安安、朱建华等同志的鼎力帮助, 付出了辛勤劳 动, 谨此致谢.

\section{4 参考文献}

[1] 唐军武,田国良,汪小勇等. 水体光谱测量与分析 I: 水面以上法. 遥感学报, 2004,8(1):37 - 44 .

[2] 宋庆君,唐军武. 黄海、东海海区水体散射特性研究. 海洋学报,2006,28(4):56 - 63 .

[3] Ma Ronghua, Tang Junwu, Dai Jinfang. Bio-optical model with optimal parameter suitable for Lake Taihu in water colour remote sensing. International Journal of Remote Sensing, 2006, 27 : 4303 - 4326.

[4] Ma Ronghua, Tang Junwu, Dai Jinfang et al. Absorption and scattering properties of water body in Lake Taihu, China: absorption. International Journal of Remote Sensing ,2006, 27 : 4275 - 4302.

[5] Lodhi M A, Rundquist D C. A spectral analysis of bottom-induced variation in the colour of Sand Hills lakes, Nebraska, USA. International Journal of Remote Sensing, 2001, 22 : 1665 - 1682.

[6] 马荣华,戴锦芳. 太湖水色遥感中叶绿素和悬浮物的高光谱分析. 见: 庄逢甘, 陈述彭主编. 中国遥感 应用协会 2005 年年会论文集. 北京: 中国宇航出版社,2005:236-245. 